Sonication of Abdominal Drains : Clinical Implications of Quantitative Cultures for the Diagnosis of Surgical Site Infection

\title{
Prieto-Borja, Laura
}

2016-08

Prieto-Borja , L , Perez-Tanoira , R , Levano-Linares , D-C , Celdran , A , Mahillo-Fernandez , I \& Esteban , J 2016 , ' Sonication of Abdominal Drains : Clinical Implications of Quantitative Cultures for the Diagnosis of Surgical Site Infection ', Surgical Infections, vol. 17 , no. 4 , pp. 459-464 . https://doi.org/10.1089/sur.2015.268

http://hdl.handle.net/10138/224228

https://doi.org/10.1089/sur.2015.268

publishedVersion

Downloaded from Helda, University of Helsinki institutional repository.

This is an electronic reprint of the original article.

This reprint may differ from the original in pagination and typographic detail.

Please cite the original version. 


\title{
Sonication of Abdominal Drains: Clinical Implications of Quantitative Cultures for the Diagnosis of Surgical Site Infection
}

\author{
Laura Prieto-Borja, Ramón Pérez-Tanoira,,2 Dennis-César Levano-Linares, ${ }^{3}$ Angel Celdrán, \\ Ignacio Mahillo-Fernández, and Jaime Esteban ${ }^{1}$
}

\begin{abstract}
Background: The use of drains in patients undergoing abdominal surgery has been a subject of debate for several decades. In this paper, the usefulness of quantitative cultures of sonicated abdominal drains for diagnosing surgical site of infection (SSI) and the association between culture results with patient outcome is evaluated.

Methods: Forty-five abdominal drainage tubes from 35 patients who underwent abdominal surgery were studied. Samples were sonicated for $5 \mathrm{~min}$, the sonicate was centrifuged, and the sediment was cultured on different media. Total bacterial counts were adjusted to the actual surface of the drainage tubing. Clinical information of the patients was reviewed retrospectively.

Results: A relation was observed between SSI and the use of drains for more than $3 \mathrm{~d}(\mathrm{p}=0.0216)$. The presence of a suspected pathogen was related to the prevalence of SSI $(p=0.035)$, complications $(p=0.013)$, and greater leukocyte count ( $\mathrm{p}=0.048$ Mann Whitney test), as well as to the use of drains for more than $3 \mathrm{~d}(\mathrm{p}=0.0386)$ and to the serous appearance of the exudates at the point of insertion of the drain $(p=0.0399)$. The sonication procedure showed a sensitivity of $50 \%$, specificity of $84.2 \%$, positive predictive value of $72.72 \%$, and negative predictive value of $66.67 \%$ in the diagnosis of SSI. The most commonly isolated group of organisms was coagulase-negative staphylococci, being present in 18 patients $(51.43 \%)$ who, however, were not associated with SSI. One or two organisms considered as pathogens were detected in 11 patients $(31.43 \%)$. The more common pathogens detected were Enterobacteriae spp. (nine patients): Enterobacter aerogenes (2), Enterobacter cloacae (1), Escherichia coli (4), Klebsiella pneumoniae (1), Morganella morganii (1); and Pseudomonas aeruginosa (five patients). Candida spp. and Enterococcus spp. were detected in one patient each one. Conclusions: The detection and quantification of organisms not present in skin microbiota after drain sonication is helpful in the diagnosis of SSI and it is associated with a worse outcome in patients. Duration of use of drainage tubes is an independent risk factor for the development of SSI.
\end{abstract}

I NTRA-OPERATIVELY, surgical drains are placed to prevent the post-operative accumulation of fluid. Pancreatic fistula and abscesses are serious and frequent complications after intra-abdominal operations [1]. Insertion of drains during surgery is the standard of care for patients with intraabdominal abscesses.

Several publications have appeared in recent years documenting the incidence and prevalence of infection-related complications in the peritoneal cavity or other locations having an implanted biomaterial [2-6]. A causal relation between the use of surgical drains and surgical site infections (SSI) has not been clearly established in the literature, and in recent years, prophylactic drainage of the peritoneal cavity has become less popular [7].

A study by Iwata et al. found that drains may be left in place for an extended period without increasing the risk of infection, even in the presence of prosthetic material; thus, the prophylactic use of antibiotics to prevent infection of

\footnotetext{
${ }^{1}$ Department of Clinical Microbiology, ${ }^{3}$ Department of Surgery, ${ }^{4}$ Department of Epidemiology, IIS-Fundación Jiménez Díaz, Madrid, Spain.

${ }^{2}$ Otorhinolaryngology-Head and Neck Surgery, University of Helsinki and Helsinki University Hospital, Helsinki, Finland.
} 
drains is unnecessary [8]. Several other studies have indicated that drains could potentially result in either SSI or an intra-abdominal infection via extra-luminal or luminal colonization of the drain by pathogens and their subsequent migration along the surface of the drain or via drained liquid, allowing bacteria access to deeper areas in the surgical space [9].

Bacterial adherence to biomaterials has been implicated as an initial step in the cascade of events leading to eventual bacterial colonization and subsequent sepsis. Thus, to counter the problem of biomaterial-associated infections, the prevention of bacterial adherence is crucial, and in recent years, research on biomaterials with antimicrobial or anti-adhesive properties has intensified [10].

Septic implant analysis is important because infected material becomes a reservoir for the pathogen. Both the diagnosis and treatment of prosthetic infections is complicated because of the development of a bacterial biofilm, wherein the bacteria often change their phenotypes to a resistant sessile form of life [11-12]. The ideal diagnostic approach to establish the presence of a pathogen requires high sensitivity and specificity of the microbiological investigation technique to confirm true infection of the drain in the context of the appropriate clinical picture. In this prospective study, we have explored the usefulness of quantitative cultures from the sonicate of abdominal drains for the diagnosis of SSI. The species and the number of colony forming units (CFU) detected in cultures have been evaluated in order to discriminate between contamination and true infection. We have also established a relation between the microbiological results and true SSI related to drains in an intra-abdominal surgery practice using a methodology with proved usefulness in the diagnosis of other kind of implantrelated infections [2-6].

\section{Patients and Methods}

\section{Samples and patients}

Removed drains of patients, who underwent routine laparotomy and laparoscopic surgeries at the Department of General Surgery at the Fundación Jiménez Díaz Hospital during March to June 2013, were included in this prospective study. They were removed under aseptic conditions when the patients were clinically stable, without post-operative intraabdominal sepsis.

The distal intra-abdominal portion of each drain was aseptically cut, collected in a sterile tube, and immediately sent to the Microbiology department.

Essentially, drains may be classified as "active" when they are connected to a suction device or "passive" when their function depends on gravity [13]. The type of drainage tubing evaluated in this study was "active." The three different types of drainage tubing in the samples studied were Jackson Pratt, Blake (both made of silicone), and Redon (made of polyvinyl chloride).

\section{Microbiological procedures}

In a biosafety cabinet, samples were introduced aseptically in $50 \mathrm{~mL}$ of sterile phosphate buffer (PBS) (pH 6.8, BioMérieux, Marcy-l'Étoile, France) in Falcon tubes. The closed Falcon tubes were then subjected to the sonication process for 5 min [14-15] in a low power sonicator $(\mathrm{Hz}=50 / 60)(\mathrm{J} . \mathrm{P}$.
Selecta, Abrera, Spain) using the protocol of Esteban et al. [11]. After that, the drainage tubing was removed and measured in order to adjust the amount of bacteria to the actual surface area of the implant.

The sonicate was centrifuged at $2500 \mathrm{xg}$ for $10 \mathrm{~min}$, and supernatant was then discharged. Sediment was re-suspended in $1.5 \mathrm{~mL}$ of PBS, vortexed, and then $10 \mathrm{mcL}$ of the suspension was inoculated into each of the following culture media: Tryptic soy 5\% sheep blood agar, chocolate agar, Schaedler 5\% sheep blood agar, MacConkey agar, and ChromID $^{\mathrm{TM}}$ Candida Agar (CAN2) for levadures (all from BbioMérieux, Marcy-ĹÉtoile, France). All media were incubated for $7 \mathrm{~d}$ at $37^{\circ} \mathrm{C}$ under different conditions: $5 \% \mathrm{CO}_{2}$ atmosphere (tryptic soy 5\% sheep blood agar and chocolate agar), normal atmosphere (MacConkey agar and ChromID Candida Agar [CAN2]), and anaerobic atmosphere (Schaedler 5\% sheep blood agar). The media were checked daily for microbial growth, and the result was expressed quantitatively in $\mathrm{CFU} / \mathrm{mL}(\mathrm{CFU} / \mathrm{mL}=\mathrm{CFU}$ on the plate $/ 10 \mathrm{mcL} \times 1000$ $\mathrm{mcL} / 1 \mathrm{~mL}=\mathrm{CFU}$ on the plate $\times 100)$.

Total bacterial counts were adjusted to the actual surface area of the drainage tubing, taking into account both the luminal and extra-luminal surface (total bacterial count $=\mathrm{CFU} /$ $\mathrm{mL} \times 1.5 \mathrm{~mL} /$ inner + outer drain surface $\left.\left[\mathrm{cm}^{2}\right]\right)$.

Isolated organisms were identified by matrix-assisted laser desorption ionization time-of-flight (MALDI-TOF) system (Vitek-MS@ BioMérieux, Marcy-l'Étoile, France). Susceptibility testing was performed using disc-plate assay according to European Committee on Antimicrobiol Susceptibility Testing (EUCAST) methodology.

\section{Clinical data}

The study was approved by the Ethical Committee of Fundación Jiménez Díaz Hospital. Clinical charts of patients whose drains were removed were reviewed. The following data were analyzed: Demographic data, type of surgical drainage, post-operative day of drain removal, post-operative complications, underlying diseases (especially diabetes mellitus, hypertension, overweight, kidney disease, respiratory disease, infectious diseases), analytical parameters, previous positive cultures, previous abdominal surgery, antibiotic treatments, and outcome. In case two or more drains of the same patient were analyzed, the following criteria were used: Only a positive culture was considered, and with two or more positive cultures, the following selection algorithm was applied: a) Only one in which some potential pathogen had been isolated was taken into account and b) with two or more positive cultures in which some potential pathogens had been isolated, the culture with greater CFU was taken into account.

Furthermore, participants were classified into two groups taking into account the presence or absence of clinical criteria of SSI [16], the latter being considered as control group. A diagnosis of SSI was made when at least one of the following was present: Purulent drainage; organisms isolated from an aseptically obtained culture of fluid or tissue from the superficial incision or in the affected organ/space; at least one of the following signs or symptoms of infection: Pain or tenderness, localized swelling, redness, or heat, and superficial incision; and diagnosis of SSI by the surgeon or attending physician [17]. The surgical incisions of the patients were classified in Class I/Clean, Class II/Clean-Contaminated, 
Class III/Contaminated, and Class IV/Dirty-Infected according to the Guideline for Prevention of Surgical Site Infection of U.S. Centers for Disease Control and Prevention (CDC) [17].

\section{Statistical analysis}

Data were analyzed using the Stata 11 software with continuous variables expressed as the mean \pm standard deviation (SD), and qualitative variables were expressed as absolute and relative frequencies.

Statistical parameters of sensitivity (Sn), specificity (Sp), positive predictive value (PPV), and negative predictive value (NPV) were calculated considering the isolation of pathogen in culture, either in presence, or in absence of clinical infection. Continuous variables were compared using Mann-Whitney U test, and qualitative variables using Fisher exact test. A $\mathrm{p}<0.05$ was considered to indicate statistical significance. Additionally, non-parametric Spearman rank correlation coefficient was used to analyze continuous variables.

\section{Results}

\section{Clinical results}

The method was tested on 45 drainage tubes from 35 patients. Among the 45 samples, there were 27 (60.0\%) Jackson Pratt, 13 (28.9\%) Blake, and 5 (11.1\%) Redon drainage tubes, of which only 24 (68.6\%) Jackson Pratt, eight Blake (22.8\%) and 3 Redon (8.6\%) drainage tubes were considered because of the assumptions made for a patient having two or more drains.

The average age of the patients included in the study was $60.14 \pm 16.55 \mathrm{y}$ and the age range was $24-88 \mathrm{y}$, including 20 males and 15 females. Participants were classified into two groups: Those with SSI $(n=16)$ and those without SSI $(n=19)$. The overall clinical data for patients with SSI and patients without SSI are summarized below in Table 1.

Table 1. Epidemiological and Clinical Results of Patients WITH SSI and Patients Without SSI

\begin{tabular}{lccc}
\hline & $\begin{array}{c}\text { SSI } \\
(\mathrm{n}=16)\end{array}$ & $\begin{array}{c}\text { Without SSI } \\
(\mathrm{n}=19)\end{array}$ & $\mathrm{p}$ \\
\hline Age (Mean \pm SD) & $57.75 \pm 12.04$ & $62,16 \pm 19,687$ & 0.371 \\
Gender (male) & $11(58.8 \%)$ & $9(47.4 \%)$ & 0.306 \\
Comorbidities & $9(56.2 \%)$ & $12(63.2 \%)$ & 0.739 \\
Complications & $13(81.2 \%)$ & $10(52.6 \%)$ & 0.152 \\
$\begin{array}{l}\text { Serous wound } \\
\quad \text { appearance }\end{array}$ & $15(93.8 \%)$ & $18(94.7 \%)$ & $\leq 1$ \\
$\begin{array}{l}\text { Tubes Jackson Pratt* } \\
\text { Tube of silicone }\end{array}$ & $13(81.2 \%)$ & $11(57.9 \%)$ & 0.167 \\
$\begin{array}{l}\text { Tube >3d } \\
\text { Surgical incision }\end{array}$ & $16(93.8 \%)$ & $17(89.5 \%)$ & 0.500 \\
$\quad$ clean** & 0 & $13(68.4 \%)$ & 0.022 \\
Antibiotic*** & $9(56.2 \%)$ & $7(36.8)$ & 0.318 \\
Fever & $4(25.0 \%)$ & $3(15.8 \%)$ & 0.497 \\
Laparotomy**** & $15(93.8)$ & $11(57.9 \%)$ & 0.016 \\
\hline
\end{tabular}

SSI = surgical site infection; $\mathrm{SD}=$ standard deviation .

* Only one tube was considered for each patient in this table; the one with the microbiological result was considered.

**According to the Guideline for Prevention of Surgical Site Infection.

***Antibiotic pre-removal of drainage.

****Laparotomy surgery compared with laparoscopy.
The analysis of clinical data indicated that 20 patients presented some kind of complication after surgery. In spite of the high number of complications, only two patients needed a re-operation, and in both cases it was an exploratory laparotomy. Only seven patients showed fever, and it was not related to SSI $(\mathrm{p}=0.497)$

A total of 21 patients presented an underlying disease, mainly diabetes mellitus, neoplastic disease, cardiovascular disease, and hepatitis (everyone with five cases) but they were not associated with surgical infections as is shown in Table 1. Other underlying diseases were kidney disease (three cases), respiratory disease (one case), and coagulopathy (one case).

Among the complications directly related to the surgical procedure $(n=14)$, the most frequent was paralytic ileus $(n=6)$, followed by surgical incision infection $(n=3)$, leakage $(\mathrm{n}=2)$, fistula $(\mathrm{n}=2)$, pancreatitis $(\mathrm{n}=1)$, and hematoma $(\mathrm{n}=1)$. Other complications included $(\mathrm{n}=15)$ hydropneumothorax $(\mathrm{n}=2)$, sepsis $(\mathrm{n}=2)$, anaemia $(\mathrm{n}=2)$, pain $(n=2)$, nosocomial pneumonia $(n=1)$, hypocalcemia $(n=1)$, hypoproteinemia $(n=1)$, bladder perforation $(n=1)$, urinary tract infection $(n=1)$, and leakage $(n=1)$. None of the mentioned complications were statistically associated with SSI.

The mean of days with tube was $6.40 \pm 4.42 \mathrm{~d}$. It was found that the presence of tube in situ for a period longer than for more of $3 \mathrm{~d}$ was associated with SSI $(\mathrm{p}=0.022)$.

The operation performed was colon $(\mathrm{n}=20)$, liver and gallbladder $(n=10)$, abdominal wall $(n=3)$, and oesophagusstomach $(n=2)$. Laparotomy surgery was performed in eight cases and laparoscopy in 27 patients. There was no statistical association between the type of the operation performed $(\mathrm{p}=0.235)$ or laparotomy surgery $(\mathrm{p}=0.595)$ and SSI.

The data did not reveal any relation between the degree of surgical incision contamination and SSI $(\mathrm{p}=0.234)$. The most frequent kind of surgical incision was clean-contaminated $(\mathrm{n}=17)$, followed by contaminated $(\mathrm{n}=11)$, dirty-infected $(n=4)$, and clean $(n=3)$.

The prophylactic antibiotic therapy taken by 16 participants (chemoprophylaxis not included) before the operation did not result in substantial differences in SSI $(p=0.318)$. The most frequent class of agents used was $\beta$-lactam antibiotics.

\section{Laboratory results}

Positive cultures were found in 37 of the 45 tubes studied. Of these, ten were monomicrobial and 27 were polymicrobial. The median of the bacterial count among positive samples was $310.6 \mathrm{CFU} / \mathrm{cm}^{2}$ (IQR $98.5-824.2 \mathrm{CFU} / \mathrm{cm}^{2}$ ). The most commonly isolated group of organisms was coagulase negative staphylococci (CNS) (26 tubes), but there was no evident relation between its presence and SSI or complications in general $(\mathrm{p} \leq 1)$. Consequently, it was regarded as a contamination because this group is the skin microbiota. On the other hand, in 11 cases, an organism different from skin microbiota was detected and was considered to be a potential pathogen (six drains with only one pathogen and five with two pathogens). The organisms were Enterobacteriae (Enterobacter aerogenes (2), Enterobacter cloacae (1), Escherichia coli (4), Klebsiella pneumonia (1), Morganella morganii (1)), Pseudomonas aeruginosa (5), Candida spp (1), and Enterococcus faecium (1). All of these organisms are known as common aetiological agents of several infections. 
Table 2. Laboratory Results of Patients with SSI and Patients without SSI

\begin{tabular}{lcrr}
\hline & $\begin{array}{c}\text { SSI } \\
(\mathrm{n}=16)\end{array}$ & \multicolumn{1}{c}{$\begin{array}{c}\text { Without SSI } \\
(\mathrm{n}=19)\end{array}$} & $\mathrm{p}$ \\
\hline CFU $>1,000$ & $9(56.2 \%)$ & $10(52.6 \%)$ & $\leq 1$ \\
Pathogen tube* & $8(50.0 \%)^{*}$ & $3(15.8 \%)^{*}$ & 0.032 \\
Concomitant culture** & $2(12.5 \%)$ & 0 & 0.202 \\
Leukocyte (Mean \pm SD) & $8,814.00 \pm 3,669.34$ & $8,288.21 \pm 3,228.66$ & 0.827 \\
CRP (Mean \pm SD) & $12.85 \pm \quad 9.35$ & $11.04 \pm$ & 5.44 \\
\hline
\end{tabular}

$\mathrm{SSI}=$ surgical site infection; $\mathrm{CFU}=$ colony forming units; $\mathrm{SD}=$ standard deviation; $\mathrm{CRP}=\mathrm{C}$ reative protein.

*Pathogen isolated from abdominal drains.

**The same pathogen isolated in a culture from blood, surgical incision exudate, or drainage fluid. It was realized only for nine patients.

Table 2 summarizes the laboratory results of patients with SSI. Only the presence of a potential pathogen was related to the incidence of SSI $(\mathrm{p}=0.035)$. As can be observed from Table 3, the isolation of pathogens from sonicated abdominal drains corresponds well with complications $(\mathrm{p} \leq 0.001)$ and greater leukocytes count $(\mathrm{p}=0.048$ Mann Whitney test). However, there was no relation between presence of a potential pathogen and $\mathrm{C}$ reactive protein (CRP) levels $(p=0.100)$, co-morbidities $(p=0.234)$, level of contamination of the operation $(\mathrm{p}=0.349)$, type of the operation $(\mathrm{p}=0.545)$, fever $(p=0.856)$, or laparotomy surgery $(p=0.674)$. The negative culture for potential pathogens showed a greater use of previous antibiotic treatment $(\mathrm{p}=0.003)$ but no statistical relation to co-morbidities $(\mathrm{p}=0.234)$. Moreover, there was a good correlation between the presence of pathogen and the extended use of drains for more than $3 \mathrm{~d}(\mathrm{p}=0.039)$ or serous appearance of the exudates at the insertion point of the drain $(\mathrm{p}=0.040)$.

In our data, CFU corresponded well with CRP levels $(\mathrm{p}=0.048$, Spearman rank correlation coefficient) but no with greater leukocytes count $(\mathrm{p}=0.183)$. The results of a statistical study comparing the outcomes between the use of a single versus two drains showed that the presence of two drains did not predict a worse outcome than that in patients having only one.

\section{Statistical parameters}

Statistical parameters relating to the usefulness of the sonication technique for the microbiological diagnosis of SSI were $\mathrm{Sn}=50 \%, \mathrm{Sp}=84.2 \%, \mathrm{PPV}=72.72 \%$, and $\mathrm{NPV}=$
$66.67 \%$. The presence of clinical infection and the isolation of a potential pathogen were the criteria considered.

\section{Discussion}

Prophylactic intra-abdominal drains are widely used in surgical practice to prevent the accumulation of fluid postoperatively [17-19]. At the present time, little information exists in the literature about their usefulness, and the use of abdominal drainage after surgery is, therefore, often dogmatic. Some surgeons have reported that their use is associated with complications as a risk of ascending infections $[13,20]$.

This paper proposes the potential usefulness of sonication as an innovative and easy technique in the microbiological diagnosis of an infection of abdominal drains. Sonication technique uses low-power ultrasound that liberate all biofilmembedded organisms from the implants, allowing a more sensitive technique for microbiological diagnosis, especially for prosthetic joint infection [21-22] and other implants [23].

Chisena et al. came up with the colonization of drains to be an element of clinical relevance in the pathogenesis of sepsis using the sonication [20]. They concluded that Staphylococcus epidermidis was the chief contaminant of abdominal drainages and their slime production was a pathogenic factor in sepsis [20]. In this context, our results show that only the presence of microorganisms that are not part of the skin microbiota from sonicated drains was associated with clinical criteria of infection, clinical complications, and a worse outcome of patients. Drains are in contact with the skin, unlike samples such as prostheses, so it is plausible to find

Table 3. Results for Negative Culture, Potential Contaminants, and Potential Pathogens for Patients $(n=35)$

\begin{tabular}{lccc}
\hline & $N C(\mathrm{n}=4)$ & $P C(\mathrm{n}=20)$ & $P P(\mathrm{n}=11)$ \\
\hline Age (Mean \pm SD) & $59.73 \pm 17.79$ & $59.79 \pm 16.67$ & $60.45 \pm 14.18$ \\
Gender (male) & $3(75.0 \%)$ & $9(45.0 \%)$ & $8(72.7 \%)$ \\
Comorbidities & $3(75.0 \%)$ & $13(65.0 \%)$ & $5(45.4 \%)$ \\
Complications & $1(25.0 \%)$ & $8(40 \%)$ & $11(100 \%)$ \\
Leukocyte (Mean \pm SD) & $8,258.81 \pm 2758.10$ & $8,066.67 \pm 3133.72$ & $9,072 \pm 3122$ \\
CRP (Mean \pm SD) & $9.30 \pm \quad 6.41$ & $12.26 \pm 7.60$ & $11.70 \pm 7.86$ \\
Antibiotic* & $1(25.9 \%)$ & $14(70.0 \%)$ & $(9.1 \%)$ \\
Fever & $2(50.0 \%)$ & $3(15.0 \%)$ & $2(18.2 \%)$ \\
Laparotomy** & $2(50.0 \%)$ & $3(15.0 \%)$ & $3(27.3 \%)$ \\
\hline
\end{tabular}

$\mathrm{NC}=$ negative culture $\mathrm{PC}=$ potential contaminant $\mathrm{PP}=$ potential pathogen.

*Antibiotic pre-removal of drain.

**Laparotomy surgery compared with laparoscopy. 
skin microbiota in drains. However, we should consider that patients with negative culture for potential pathogens (negative culture $[\mathrm{NC}]$ and potential contaminant [PC] groups) have received, in a greater proportion, previous antibiotic treatment to remove of the tube. It would reduce the isolation of potential pathogens from the tube allowing the growth of bacteria from the skin.

In some cases, common colonizers of the gastrointestinal tract of healthy individuals were recovered from the tubes. For example, Enterococcus faecium was recovered in mix culture with bacteria from skin in one case. This microorganism has emerged as an important cause of hospitalassociated infections. The effects of antibiotics on the gut microbiota and on colonization with antibiotic-resistant enterococci are highlighted, including how enterococci benefit from the antibiotic-mediated eradication of gram-negative members of the gut microbiota [24-26].

As observed, elevated values of CFU correspond well with elevated values of CRP, but the significance of this finding is not clear because CRP is an acute phase reactant that is considered an inflammation marker; thus, its increase does not mean infection necessarily. Moreover, the association of high CFU and elevated CRP values could suggest the presence of an infection in this setting. Thus, unlike in urine cultures where counts $>10^{5} \mathrm{CFU} / \mathrm{mL}$ are related to clinical infection [27-28], in this work, a similar degree of CFU is not evident. However, taking into account that the presence of a pathogen is the parameter related to clinical infection, we could recommend the minimum count in which a pathogen is detected $(1,500 \mathrm{CFU} / \mathrm{mL})$ as the threshold colony count that indicates that the infection is clinically relevant.

Our attention was focused not only on the use of sonication for diagnosis of abdominal drains infection but also on the consequences of long duration of drains in the outcome of patients. As mentioned earlier, we have found that the presence of the tube for more than $3 \mathrm{~d}$ was related to the positive culture for potential pathogens and to a clinical diagnosis of SSI. The results are in good agreement with those of other studies, such as Kawai et al. [29]. The latter concluded in a prospective, non-randomized study that drain removal on post-operative day four was shown to be an independent factor in reducing the incidence of complications, including intra-abdominal infections. Moreover, serous appearance of the exudates at the insertion point of the drain was also related to the detection of a pathogen. In light of our results and the results of Vecchio et al. [13] and Fan et al. [30], we dare to recommend that implants should be removed as soon as appearance at the insertion point becomes serous. Early removal of the drains plays an important role in diminishing incidence of abdominal infections $[13,30]$.

Further research is needed to understand better the role of the type of drain tube or the composition of the tube in the incidence of SSI. Our results demonstrate that it was related neither to SSI nor solely to the presence of a pathogen, but it must be noted that the small number of tubes made of polyvinyl chloride (Redon) is a limitation of the study result (11\% of all tubes).

Furthermore, the patients having two drains did not present a worse outcome than patients having only one, a result that is consistent with the study of Shrikhande et al. [31]. Again, these results are not conclusive because of the small number of evaluated patients in the study.
Further, it needs to be noted that although differences in the counts if mixed cultures were large enough to consider them as reliable measures, it is difficult to know the true total CFU of each species. Each species has a different speed of growth and in turn can affect that of the other species, a fact that may be observed as a limitation of this study.

Future work of our laboratory will investigate the results from both surgical incision and blood cultures, and these will then be compared with those obtained by sonication of drains in the same patient.

From our research, it is possible to conclude that duration of use of drains is an independent risk factor for the development of SSI, and they should be removed as soon as possible to avoid contamination by microorganisms. The presence of an abdominal drain can lead to persistent gut germs that without the existence of the foreign body could be eliminated by the peritoneum.

The positive culture from the drain for potential pathogens in patients without clinical should be treated empirically when there are risk factors, as is a blood withdraw after cholecystectomy advanced cholecystitis or immunosuppression. The findings lend support to the idea that sonication of drains could be useful in the diagnosis of SSI. More tests will be needed to verify the significance of our result with data of cultures from sonicated drains in similar and other surgical settings.

\section{Acknowledgments}

This work was supported by a grant from the Spanish Ministry of Science and Technology (FUNCOAT CSD 20080023) and funds from the Surgery Department of the Fundación Jiménez Díaz University Hospital.

\section{Author Disclosure Statement}

No conflict of interest for any of the authors regarding this manuscript.

\section{References}

1. Sano T, Sasako M, Yamamoto S, et al. Gastric cancer surgery: Morbidity and mortality results from a prospective randomized controlled trial comparing D2 and extended para-aortic lymphadenectomy-Japan Clinical Oncology Group study 9501. J Clin Oncol 2004;22:2767-2773.

2. Perez-Tanoira R, Isea-Pena MC, Celdran A, et al. Bacterial adherence to different meshes used in abdominal surgery. Surg Infect (Larchmt) 2014;15:90-93.

3. Burger JW, Luijendijk RW, Hop WC, et al. Long-term followup of a randomized controlled trial of suture versus mesh repair of incisional hernia. Ann Surg 2004;240:578-583.

4. Molina-Manso D, del Prado G, Ortiz-Perez A, et al. In vitro susceptibility of Staphylococcus aureus and Staphylococcus epidermidis isolated from prosthetic joint infections. J Antibiot (Tokyo) 2012;65:505-508.

5. Katsikogianni M, Missirlis YF. Concise review of mechanisms of bacterial adhesion to biomaterials and of techniques used in estimating bacteria-material interactions. Eur Cell Mater 2004;8:37-57.

6. Darouiche RO. Device-associated infections: A macroproblem that starts with microadherence. Clin Infect Dis 2001;33:1567-1572.

7. Puleo FJ, Mishra N, Hall JF. Use of intra-abdominal drains. Clin Colon Rectal Surg 2013;26:174-177. 
8. Iwata N, Kodera Y, Eguchi T, et al. Amylase concentration of the drainage fluid as a risk factor for intra-abdominal abscess following gastrectomy for gastric cancer. World J Surg 2010;34:1534-1539.

9. Vilar-Compte D, Mohar A, Sandoval S, et al. Surgical site infections at the National Cancer Institute in Mexico: A case-control study. Am J Infect Control 2000;28:14-20.

10. Guo W, Andersson R, Odselius R, et al. Phospholipid impregnation of abdominal rubber drains: Resistance to bacterial adherence but no effect on drain-induced bacterial translocation. Res Exp Med (Berl) 1993;193:285-296.

11. Esteban J, Gomez-Barrena E, Cordero J, et al. Evaluation of quantitative analysis of cultures from sonicated retrieved orthopedic implants in diagnosis of orthopedic infection. J Clin Microbiol 2008;46:488-492.

12. Perez-Jorge C, Conde A, Arenas MA, et al. In vitro assessment of Staphylococcus epidermidis and Staphylococcus aureus adhesion on $\mathrm{TiO}(2)$ nanotubes on $\mathrm{Ti}-6 \mathrm{Al}-4 \mathrm{~V}$ alloy. J Biomed Mater Res A 2012;100:1696-1705.

13. Vecchio R, Intagliata E, Marchese S, et al. Surgical drain after open or laparoscopic splenectomy: Is it needed or contraindicated? G Chir 2015;36:101-105.

14. Kobayashi N, Bauer TW, Tuohy MJ, et al. Brief ultrasonication improves detection of biofilm-formative bacteria around a metal implant. Clin Orthop Relat Res 2007;457: 210-213.

15. Perez-Tanoira R, Garcia-Pedrazuela M, Hyyrynen T, et al. Effect of S53P4 bone substitute on staphylococcal adhesion and biofilm formation on other implant materials in normal and hypoxic conditions. J Mater Sci Mater Med 2015;26: 239.

16. Mangram AJ, Horan TC, Pearson ML, et al. Guideline for Prevention of Surgical Site Infection, 1999. U.S. Centers for Disease Control and Prevention (CDC). Hospital Infection Control Practices Advisory Committee. Am J Infect Control 1999;27:97-132.

17. Bassi C, Molinari E, Malleo G, et al. Early versus late drain removal after standard pancreatic resections: Results of a prospective randomized trial. Ann Surg 2010;252:207-214.

18. Buchler MW, Friess H. Evidence forward, drainage on retreat: Still we ignore and drain!? Ann Surg 2006;244:8-9.

19. Yeo CJ. Pancreatic surgery 101: Drain, no drain, early drain removal, or late drain removal. What are the data? Where do we go from here? Ann Surg 2010;252:215-216.

20. Chisena S, Marconato R, Cantoni G, et al. [Importance of Staphylococcus epidermidis in the bacterial colonization of abdominal drains in surgical patients]. Minerva Chir 1991;46: 269-272.

21. Evangelopoulos DS, Stathopoulos IP, Morassi GP, et al. Sonication: A valuable technique for diagnosis and treatment of periprosthetic joint infections. ScientificWorldJournal 2013;2013:375140.

22. Trampuz A, Piper KE, Jacobson MJ, et al. Sonication of removed hip and knee prostheses for diagnosis of infection. N Engl J Med 2007;357:654-663.

23. Malone PC. The physiology of intestinal oxygenation and the pathophysiology of intestinal ileus. Med Hypotheses 1987;22:111-57.

24. Pasticci MB, Baldelli F, Malincarne L, et al. Vancomycinresistant Enterococcus faecium osteoarthritis following Staphylococcus aureus hip infection. Orthopedics 2005;28: 1457-1458.

25. Montealegre MC, Singh KV, Murray BE. Gastrointestinal Tract Colonization Dynamics by Different Enterococcus faecium Clades. J Infect Dis 2015 [Epub ahead of print].

26. Arias CA, Murray BE. The rise of the Enterococcus: Beyond vancomycin resistance. Nat Rev Microbiol 2012;10: 266-278.

27. Rushton HG. Urinary tract infections in children. Epidemiology, evaluation, and management. Pediatr Clin North Am 1997;44:1133-1169.

28. Kwon JH, Fausone MK, Du H, et al. Impact of laboratoryreported urine culture colony counts on the diagnosis and treatment of urinary tract infection for hospitalized patients. Am J Clin Pathol 2012;137:778-784.

29. Kawai M, Tani M, Terasawa H, et al. Early removal of prophylactic drains reduces the risk of intra-abdominal infections in patients with pancreatic head resection: Prospective study for 104 consecutive patients. Ann Surg 2006; 244:1-7.

30. Fan Y, Ren J, Wu X, et al. [Risk factors of surgical site infection in definitive surgery of intestinal fistulas]. Zhonghua Wei Chang Wai Ke Za Zhi 2015;18:646-650.

31. Shrikhande SV, Barreto SG, Shetty G, et al. Post-operative abdominal drainage following major upper gastrointestinal surgery: Single drain versus two drains. J Cancer Res Ther 2013;9:267-271.

Address correspondence to:

Dr. Ramón Pérez-Tanoira

Otorhinolaryngology-Head and Neck Surgery

University of Helsinki

and Helsinki University Hospital

Haartmaninkatu 4E, 00029 HUS

Helsinki, Finland

E-mail: ramontanoira@hotmail.com 